\title{
EXPERT REVIEWS
}

\section{IL-15 modulates the effect of retinoic acid, promoting inflammation rather than oral tolerance to dietary antigens}

Expert Rev. Gastroenterol. Hepatol. 5(3), 315-317 (2011)

\section{Eduardo Arranz ${ }^{+1}$ and Jose A Garrote ${ }^{1,2}$}

'Mucosal Immunology Laboratory, Instituto de Biologia y Genetica Molecular (IBGM), University of Valladolid-CSIC, Valladolid, Spain ${ }^{2}$ Molecular Biology Laboratory, Hospital Universitario Rio-Hortega Valladolid, Spain

${ }^{+}$Author for correspondence:

Tel.: +34983184843

earranz@med.uva.es

Evaluation of: DePaolo RW, Abadie V, Tang F et al. Co-adjuvant effects of retinoic acid and IL-15 induce inflammatory immunity to dietary antigens. Nature 471(7337), 220-224 (2011).

The physiological immune response in the intestine against dietary proteins and commensal flora is characterized by regulatory mechanisms (tolerance) that prevent harmful consequences. Intestinal dendritic cells (DCS) have a central role in the development of immunosuppressive regulatory $T$ cells owing to their ability to produce TGF- $\beta$ and retinoic acid (RA). However, the article under evaluation shows an unexpected effect of RA - that of promoting a proinflammatory phenotype in intestinal DCs involved in the generation of inflammatory immune responses to dietary antigens. By using a double transgenic murine model that resembles human celiac disease, it was demonstrated that RA synergizes with IL-15 in promoting the breakdown of gluten tolerance and the development of enteropathy. The tissue microenvironment modulates DC function, and immune therapies that are based on RA aiming to restore oral tolerance should be used with caution because the presence of IL-15 (and/or other proinflammatory cytokines) may have undesirable effects.

KeYworDs: celiac disease $\bullet$ dietary antigens $\bullet$ IL-15 $\bullet$ inflammation $\bullet$ intestine $\bullet$ retinoic acid $\bullet$ tolerance

In the article under evaluation, the authors studied the in vitro effect of IL-15 on the differentiation of inducible Treg cells [1]. Celiac disease is a good example of a case in which a breakdown of oral tolerance to dietary gluten leads to the development of gluten-related enteropathy, and IL-15 is overexpressed in the intestine of patients with celiac disease [2]. By using IL-15-stimulated intestinal dendritic cells (DCs; from mesenteric lymph nodes [MLNs]), the authors demonstrated that the generation of Treg cells is impaired compared with nonstimulated controls, and that this is mediated by a direct effect of IL-15 on DCs, but not on $\mathrm{T}$ cells. These results were confirmed in vivo by feeding transgenic mice that overexpress IL-15 in the intestinal lamina propria and MLN with ovalbumin (OVA), a known model antigen used to study oral tolerance. OVA-fed transgenic mice demonstrated a significantly decreased number of OVA-specific Treg cells in MLN, as compared with the OVA-fed wild-type mice. Moreover, the addition of retinoic acid (RA) had an even greater negative effect on the generation of Treg cells in the OVA-fed transgenic mouse model.

In order to further clarify the mechanisms underlying the effects of IL-15 and RA, the authors used IL-15-stimulated splenic DCs (which unlike MLN DCs, do not produce RA), and found that IL-15 and RA cooperate in the DC-mediated production of the proinflammatory cytokines IL-12p70 and IL-23. The negative effect of IL-15 on Treg cell differentiation occurs in parallel with the induction of a T helper 1 (Th1) response (which is known to depend on IL-12), as observed by in vitro and in vivo experiments. Moreover, RA cooperates with IL-15 in promoting the development of effector T cells, and, together with the presence of either IL-12p70 or IL-6, will lead to the polarization towards a Th1 or Th17-type response, respectively. The proinflammatory effects of IL-15 (increased DC-mediated production of IL-12p70 and IL-23, and impaired generation 
of Treg cells) disappear when neutralizing anti-IL-15 antibodies are used. The synergistic effect of IL-15 and RA in promoting a proinflammatory DC phenotype involved the activation of the $J N K$ signaling pathway in a dose-dependent manner.

Focusing on the pathogenesis of celiac disease, a food protein intolerance characterized by the induction of a gluten-specific inflammatory Th1 response to dietary gluten [3], the authors tried to explain why up to $40 \%$ of the population carries the HLA-DQ2 or HLA-DQ8 molecules associated with disease susceptibility, but only $2 \%$ of these develop a gluten-sensitive enteropathy. They hypothesized that the upregulation of IL-15 in the intestinal lamina propria of celiac disease patients may be responsible for the breakdown of tolerance to gluten. Using double transgenic mice (by crossing $I L-15$ transgenic mice with humanized $H L A-D Q 8$ transgenic mice), they found that following gluten feeding, these mice developed features of early-stage celiac disease: glutenspecific IFN- $\gamma$-producing $T$ cells, antigliadin and anti-TG2 antibodies, and intraepithelial lymphocytosis, but no villous atrophy. Moreover, the induction of inflammatory responses (Th1 cells and antigliadin antibodies) was dependent on and promoted by RA.

\section{Discussion}

The mucosal immune system is characterized by the negative regulation of the immune responses to dietary antigens and the commensal flora. The underlying mechanism, known as oral tolerance [4], depends on the properties of a unique population of mucosal CD103 ${ }^{+}$DCs with the ability to secrete TGF- $\beta$ and RA, which promote the development of immunosuppressive Treg cells [5]. RA has little or no effect on its own, but acting synergistically with TGF- $\beta 1$ promotes the induction of Treg cells at high concentration, although at very low concentration Th17 cells are induced [6].

This article demonstrates that IL-15 promotes the production of the proinflammatory cytokines IL-12 and IL-23 by DCs, and reduces the ability of these cells to drive the generation of Tregs, at the same time that it induces Th1-type responses to dietary proteins. Moreover, RA should be considered not just a factor involved in oral tolerance as previously thought. Its effects depend on the intestinal microenvironment, and local factors would determine whether RA activates DCs towards a proinflammatory phenotype and the induction of immune responses against dietary antigens (by synergizing with IL-15 or IL-6, and the development of Th1 or Th17 cells, respectively), or the prevention of these responses (by cooperating with TGF- $\beta$ in Treg cell differentiation). This may be relevant for new immunomodulatory therapies aiming to restore oral tolerance in intestinal inflammatory disorders and based on the use of RA. These strategies should be used with caution because, particularly in patients suffering inflammatory disorders, including those affecting the intestine, the outcome may be not what it is expected when IL-15 and/or other proinflammatory cytokines are present.

The authors suggested that "there are no unconditional suppressive factors, and that integration of tissue and exogenous signals determine the class of the immune response...", and this is perhaps the relevant point: the ability of the tissue environment to modulate the phenotype and function of DCs may change depending on the context, with unwanted results. Future studies should aim to confirm these results in humans, identifying possible differences between intestinal DCs in humans and animal models, such as mice, as well as studying the effects of other factors or cytokines (e.g., IL-4 or -5) in the same context. Moreover, it would be very interesting to know how and how long these proinflammatory DCs can be reprogrammed for, and, if the reprogramming process were to be performed ex vivo, how the in vivo conditions would affect the phenotype of these DCs.

Celiac disease is a chronic inflammatory intestinal disorder caused by an inappropriate immune response to dietary gluten proteins (prolamines) from cereals in genetically predisposed (HLA-DQ2 or $-D Q 8)$ individuals [2]. The disease is the result of the breakdown of tolerance to gluten proteins and the induction of an inflammatory Th1 response characterized by high levels of IFN- $\gamma$ and other proinflammatory cytokines [3]. These glutenreactive $\mathrm{CD}_{4}^{+} \mathrm{T}$ cells from the intestinal lamina propria recognize gluten peptides modified by the enzyme tissue transglutaminase (TGt) in the context of HLA-DQ2 or -DQ8 molecules presented by local antigen-presenting cells (such as DCs). Moreover, gluten also induces the expression of IL-15 by enterocytes, mediating cytotoxicity against the intestinal epithelium [7].

What this article demonstrates by using a double transgenic mouse model (the result of crossing humanized $H L A-D Q 8$ and IL-15 transgenic mice), is that IL-15 and RA cooperate in the breakdown of tolerance to dietary gluten owing to the IL-15mediated production of proinflammatory cytokines by DCs, which leads to the induction of an aberrant inflammatory Th1 response to gluten proteins. This may explain why only a small percentage of $H L A-D Q 2 /-D Q 8$-positive individuals develop the gluten-associated enteropathy. Moreover, under inflammatory conditions (such as overexpression of IL-15, as occurs in celiac disease), RA acts as a co-adjuvant in the induction of the inflammatory T-cell-mediated response to gluten. The results also support the idea that IL-15 should be included as a target in any future therapeutic strategy for celiac disease and, perhaps, for other intestinal inflammatory disorders.

Finally, the authors proposed that the double transgenic $H L A-D Q 8$ and $I L-15$ mice may be the long-awaited model for the study of the physiopathology of the early stages of celiac disease. No doubt this is an important step forward; however, the model does not reproduce the complete pathogenesis of celiac disease, but the early events of the disease perhaps related to the activation of the adaptive arm of the immune response to gluten proteins. This should be taken into account when extrapolating the results. For example, the model is characterized by overexpression of IL-15 in the lamina propria, but before epithelial damage and villous atrophy takes place, though intraepithelial lymphocytosis is observed. In human celiac disease, at least part of IL-15 synthesis depends on the enterocytes under stress directly induced by gluten peptides becoming the target of cytotoxic mechanisms. Actually, the sensitivity to IL-15 in human celiac disease may be increased [8]. Another difference is related to IL-12 production: the correlation between IL-15 and IL-12 reported in the article is not strong enough (with just one case showing high IL-12 expression). 
Moreover, it has been previously reported that IL-12 is absent from the intestine of celiac disease patients [3,9], and the relevance of the $I L-12 A$ gene for celiac disease predisposition is not yet known.

\section{Five-year view}

The article under evaluation is an important step forward in the direction of deciphering the mechanisms underlying oral tolerance to dietary proteins and the commensal flora, and it shakes some preconceived ideas, particularly regarding the role of RA and other local microenvironmental factors. There is a great need to clarify and understand these mechanisms, although this will take some time and does not seem to be round the corner yet. The article discussed is relevant to celiac disease, a model where the upregulation of IL-15 in the intestine, and the co-adjuvant effect of RA may lead to a breakdown of oral tolerance and the development of a gluten-related enteropathy. However, the results shown here have to be validated in humans. It is conceivable that an animal model with the ability of reproducing the complete physiopathology of celiac disease will be available in a few years. This (still) missing model will be very useful for the study of dietary protein-related disorders. An important task ahead is to find out how intestinal immune homeostasis is maintained, and how conditioning factors, such as RA in the context of cytokines, determine the acquisition of a tolerogenic or proinflammatory phenotype in DCs. Translating this knowledge to design immunomodulatory strategies based on DC reprogramming will be a challenge. Finally, the study underlines the point that IL-15 should also be a target in future intestinal anti-inflammatory therapies.

\section{Financial \& competing interests disclosure}

The authors have no relevant affiliations or financial involvement with any organization or entity with a financial interest in or financial conflict with the subject matter or materials discussed in the manuscript. This includes employment, consultancies, honoraria, stock ownership or options, expert testimony, grants or patents received or pending, or royalties.

No writing assistance was utilized in the production of this manuscript.

\section{Key issues}

- Intestinal immune responses to dietary proteins and commensal flora are strictly controlled, and dendritic cells (DCs) have a central role in this process.

- By producing TGF- $\beta$ and retinoic acid (RA), intestinal DCs drive the development of Treg cells.

- IL-15 reduces the ability of DCS to induce the generation of Treg cells and, unexpectedly, RA synergizes with IL-15 in reducing the number of Treg cells.

- In a double transgenic mouse model partially reproducing human celiac disease, IL-15 may be responsible for the breakdown of tolerance to dietary gluten.

- In this model, RA cooperates with IL-15 to induce DC-mediated production of proinflammatory cytokines (IL-12 and IL-23) and a Th1 response to gluten.

- Therefore, RA is not only a mediator of tolerance (as previously thought), but in the presence of other cytokines may activate DCs towards a proinflammatory phenotype, and the induction of specific proinflammatory Th1 responses.

- These results should be confirmed in humans, and possible differences between mouse and human intestinal DCs taken into account.

- Future therapies aiming to restore tolerance should be used with caution, particularly in patients suffering inflammatory disorders.

- These new anti-inflammatory strategies may include IL-15 as one of the targets through the use of neutralizing antibodies.

\section{References}

Papers of special note have been highlighted as:

- of interest

-• of considerable interest

$\checkmark$ DePaolo RW, Abadie V, Tang F et al. Co-adjuvant effects of retinoic acid and IL-15 induce inflammatory immunity to dietary antigens. Nature 471(7337), 220-224 (2011).

- First description of the synergistic effect of retinoic acid (RA) and IL-15 in the induction of a proinflammatory response to dietary antigens, and relevance of this in celiac disease.

2 Jabri B, Sollid LM. Mechanisms of disease: immunopathogenesis of celiac disease. Nat. Clin. Pract. Gastroenterol. Hepatol. 3, 516-525 (2006).

- Review of the mechanisms triggered by the ingestion of dietary gluten in patients with celiac disease, based on the activation of $T$ cells within the intestinal mucosa.

3 Nilsen EM, Lundin KE, Krajci P et al. Gluten specific, HLA-DQ restricted T cells from coeliac mucosa produce cytokines with Th1 or Th0 profile dominated by interferon $\gamma$. Gut 37, 766-776 (1995).

$\checkmark 4$ Faria AM, Weiner HL. Oral tolerance. Immunol. Rev. 206, 232-259 (2005).

$\checkmark 5$ Coombes JL, Siddiqui KR, Arancibia-Cárcamo CV et al. A functionally specialized population of mucosal CD103 ${ }^{+} \mathrm{DCs}$ induces Foxp3 ${ }^{+}$ regulatory $T$ cells via a TGF $\beta$ and retinoic acid-dependent mechanism. J. Exp. Med. 204, 1757-1764 (2007).

- Reports that under normal/ physiological conditions, intestinal dendritic cells show the ability of inducing the generation of
Treg cells, and RA has a central role in this process.

-6 Strober W. Vitamin A rewrites the ABC of oral tolerance. Mucosal Immunol. 1, 92-95 (2008).

7 Mention JJ, Ben Ahmed M, Begué B et al. Interleukin 15: a key to disrupted intraepithelial lymphocyte homeostasis and lymphomagenesis in celiac disease. Gastroenterology 125, 730-745 (2003).

$>_{8}$ Bernardo D, Garrote JA, Allegretti Y et al. Higher constitutive IL-15RA expression and lower IL-15 response threshold in coeliac disease patients. Clin. Exp. Immunol. 154, 64-73 (2008).

$\rightarrow$ León JA, Garrote JA, Blanco-Quirós A et al. Interleukin 18 maintains a longstanding inflammation in celiac disease patients. Clin. Exp. Immunol. 146, 479-485 (2006). 